\title{
Flip-teaching aplicado al Inglés para la Gestión Empresarial: una nueva experiencia
}

Cristina Pérez Guillot ${ }^{\mathrm{a}}$ y Asunción Jaime Pastor ${ }^{\mathrm{b}}$

aDto. Lingüística Aplicada -UPV, mcperez@upv.es y boto. Lingüística Aplicada -UPV, ajaime@upv.es

\begin{abstract}
Language learning requires an interaction between the students and the teacher in the classroom that allows for the actual practice of the communicative aspects of the language.

Given the small number of language courses and credits offered in the degrees' programs and the UPV requirements of a B2 level of language competence for the award of Bachelor's degrees, the introduction of flipteaching in the English courses helps to significantly optimize teaching resources and to better use the time available in the classroom (Bergmann, Obermyer y Willie 2011, Stone 2012).

In this paper we present a proposal for the implementation of flip-teaching in the course of English for Business Management (IGE)-B1 level, as an alternative to conventional teaching and learning methodologies. This approach allows students to review the theoretical aspects at home, through easily available technological media, such as PoliformaT or the internet, and therefore optimize the face-to-face classroom time, so necessary in teaching languages.

With this experience we expect to foster the students' motivation as they will have the opportunity of developing real communicative skills and strategies, thus improving their performance and language competence. In this way students will be better prepared to meet the UPV language reqirements for graduating and eventually improving their opportunities in the international labor market.
\end{abstract}

Keywords: Flip-teaching, Business English, TIC, student-oriented teaching, active learning 
Flip-teaching aplicado al Inglés para la Gestión Empresarial: una nueva experiencia

\begin{abstract}
Resumen
El aprendizaje de idiomas necesita una interacción entre los alumnos y el profesor en la clase que permita la práctica real de los aspectos comunicativos de la lengua.
\end{abstract}

Dado el escaso número de créditos en las titulaciones de grado dedicado a los idiomas y las exigencias de la UPV de un nivel B2 de competencia lingüística para graduarse, la introducción de flip-teaching en los programas de las asignaturas de inglés sin duda permiten optimizar los recursos y aprovechar el tiempo disponible en el aula (Bergmann, Obermyer y Willie 2011, Stone 2012).

En este trabajo presentamos una propuesta de utilización de esta metodología en la clase de Inglés para la Gestión Empresarial (IGE) nivel B1, como alternativa a la enseñanza tradicional. Se trata de combinar la posibilidad de que el alumno estudie los aspectos teóricos previamente, a través de distintos medios, como la plataforma Poliformat o internet y, de este modo, optimizar el tiempo presencial en el aula tan necesario en la enseñanza de idiomas.

Con esta iniciativa esperamos fomentar la motivación del estudiante al poder utilizar todo el tiempo presencial en la práctica real de la lengua cono lo que se logrará mejorar su rendimiento y competencia comunicativa y, de este modo, sentar la bases y afianzar sus conocimientos para poder alcanzar el nivel B2, imprescindible no solo para graduarse sino para también para acceder al mercado laboral internacional.

Palabras clave: enseñanza inversa, inglés para los negocios, TIC, aprendizaje centrado en el alumno, aprendizaje activo

\title{
1. Introducción
}

La creación del Espacio Europeo de Educación Superior (EEES) ha supuesto cambios radicales en la formación universitaria y ha evidenciado la necesidad de desarrollar políticas lingüísticas que permitan el intercambio de conocimiento y una mayor movilidad entre instituciones (Berlin Declaration, 2001; European Language Council, 2005; Haug y Vilalta, 2011).

Pero para lograr dichos objetivos es necesario que los participantes en este proceso posean un buen nivel real de competencia lingüística, por lo que el conocimiento de idiomas se ha convertido en pieza clave de integración en la nueva Europa del conocimiento: 'La globalización del empleo y la internacionalización de los nuevos puestos de trabajo

(c)) EY-NC-ND 2015, Universitat Politècnica de València Congreso IN-RED (2015) 
derivados de la incorporación de nuestro país en la Sociedad basada en el Conocimiento ha provocado que en el momento de adecuación de nuestros estudios al EEES se consideraran de forma especial la adquisición de conocimientos, habilidades y competencias de internacionalización con el fin de preparar a nuestros futuros egresados para un mundo cada vez más globalizado a nivel de empleo’ (EIU, 2011).

La Universidad Politécnica de Valencia (UPV), comprometida con el Proceso de Bolonia y consciente de la importancia de ofrecer a sus egresados una formación sólida y competitiva que les permita abordar los difíciles retos del nuevo mercado laboral, ha considerado conveniente exigir un nivel B2 de competencia lingüística en sus nuevos grados: 'para la obtención del título de Grado el alumno deberá acreditar el conocimiento de una lengua extranjera, preferentemente el inglés, con un nivel correspondiente a B2’ (Documento Marco UPV, 2008).

No obstante, aunque todas las nuevas titulaciones incluyen alguna asignatura de idiomas en sus planes de estudio, los créditos ofertados son insuficientes para alcanzar el nivel B2 de competencia exigido. $\mathrm{Si}$ a esto añadimos que el aprendizaje de idiomas necesita una interacción entre los alumnos y el profesor en la clase que permita la práctica real de los aspectos comunicativos de la lengua, resulta evidente la importancia de utilizar métodos de aprendizaje que optimicen el escaso tiempo disponible de formación presencial. Y en este sentido, la enseñanza inversa o flip teaching despunta como una alternativa prometedora a los sistema de enseñanza tradicionales (Fulton, 2012, Sams y Bergmann, 2013, Lasry et al., 2014).

La metodología de flip teaching consiste en emplear el tiempo disponible del estudiante fuera del aula para realizar determinados procesos de aprendizaje que tradicionalmente se hacen en clase para potenciar y facilitar la adquisición y práctica del conocimiento (Sánchez, 2013).

Esta forma de trabajo parte de la filosofía de aprovechar espacios de interacción dentro del aula para obtener el máximo provecho del profesor en los momentos en los que éste es más necesario, es decir, cuando el discente requiere aplicar la teoría (Demetry, 2010, Rincón, 2015).

La clave radica en que fuera del aula se pueda acceder al contenido dispuesto principalmente de manera audiovisual, desarrollándose un tema para posteriormente de manera presencial y en conjunto con los demás compañeros y el profesor, se puedan trabajar los contenidos de forma más práctica y dinámica (Gutiérrez et al., 2013).

Con la clase invertida se cambia el modelo de trabajo y se invierten los papeles de una clase tradicional gracias al apoyo de las tecnologías de la información y la comunicación (TIC). La exposición del profesor se sustituye por una serie de materiales en línea, que pueden ser 
vídeos, lecturas, etc. que el alumno puede consultar y visualizar las veces que necesite y que incluyen el contenido teórico y procedimental de una materia. El tiempo de clase, por su parte, se dedica a actividades prácticas en las que el profesor interviene como guía (Sánchez et al., 2014) La implementación de esta nueva metodología implica un cambio de paradigma ya que el docente pasa de ser el poseedor del saber a un guía y facilitador en el proceso de aprendizaje, un mediador entre el conocimiento y el alumnado para que el alumno, aparte de adquirir saberes, aprenda a aprenderlos por sí mismo. Debe buscar el máximo aprovechamiento de los nuevos recursos y de superar sus obstáculos y problemas (Sánchez et al., 2014).

En este trabajo presentamos una propuesta de utilización de esta metodología en la clase de Inglés para la Gestión Empresarial (IGE) nivel B1 de la Facultad de Administración de Empresas (FADE), como alternativa a la enseñanza tradicional.

\section{Objetivos}

La propuesta que presentamos se implementará el próximo curso académico 2015-16 para la asignatura de Inglés para la Gestión Empresarial (IGE) nivel B1 ofertada en el 3er año del grado de Administración y Gestión de Empresas de la Facultad de Administración de Empresas (FADE), y que tiene una carga docente de 4,5 créditos.

Nuestra propuesta combina la enseñanza tradicional con la metodología flip-teaching, ofreciéndole al alumno la posibilidad de practicar en casa aspectos léxicos, gramaticales y discursivos a través de distintos tipos de materiales, tanto de elaboración propia subidos a la plataforma PoliformaT como actividades y material de lectura y audiovisual disponible en el libro de texto o en internet.

Una característica de los cursos de idiomas es la diversidad de niveles de los estudiantes que se matriculan en nuestras asignaturas de inglés. La introducción de la enseñanza inversa, pues, permitirá a los alumnos trabajar a su propio ritmo según sus conocimientos (Shapiro, 2013) así como planificar la forma de abordar el material suministrado de acuerdo con sus intereses personales.

Con ello conseguiremos incrementar la motivación de los estudiantes y mejorar su rendimiento, poniendo las tecnologías al servicio del aprendizaje y fomentando el desarrollo de estrategias de autoaprendizaje que les serán de gran utilidad en su formación a lo largo de la vida.

De este modo, podremos optimizar el escaso tiempo presencial en el aula tan necesario en la enseñanza de idiomas y utilizarlo para mejorar las distintas macrodestrezas lingüísticas y 
para practicar estrategias comunicativas mediante una mayor interactividad entre profesor y alumno simulando situaciones que se aproximen a la vida real.

\section{Aplicación del Flip-teaching a la asignatura de IGE}

La asignatura de Inglés para la Gestión Empresarial (IGE) se imparte en el primer cuatrimestre de tercero de grado, con una carga docente de 4,5 créditos, de los cuales 1,3 créditos corresponden a Teoría de Aula (TA), 1 crédito a Teoría de Seminario (TS) y 2,2 créditos a Prácticas de Laboratorio (PL).

El próximo curso académico, la asignatura se ofrecerá a dos grupos de alumnos, uno los cuales se impartirá de forma presencial o convencional, y funcionará en paralelo con el nuevo grupo en el que se pretende implementar la metodología de flip-teaching. Común a ambos grupos serán las Prácticas de Laboratorio, que constan de una sesión semanal presencial de 2 horas. Ahora bien, mientras los créditos correspondientes a la TA y TS suponen una carga presencial de 1:30 horas a la semana en el caso del grupo convencional, en el nuevo grupo on-line, los 1,3 créditos correspondientes a la Teoría de Aula más 1 crédito de Teoría de Seminario los realizará el alumno en su casa aplicando el aprendizaje inverso y la carga presencial se reducirá a 50 minutos cada dos semanas, sesión que servirá para aclarar dudas concretas, y para repasar y consolidar el material que los alumnos ya han estudiado en la fase de autoestudio.

La asignatura está dividida en 5 bloques temáticos: Human resources, Marketing, Entrepreneurship, Business abroad, Change y Customer relations. Para cada uno de dichos bloques se ha elaborado una sesión de flip teaching con actividades en las que se alternan lecturas de textos de Inglés para los negocios, con el fin de que el alumno amplíe su vocabulario específico, explicaciones gramaticales de algunos aspectos esenciales de la lengua inglesa, así como material de lectura adicional, vídeos y ejercicios gramaticales complementarios que sirvan para fomentar el debate y la puesta en práctica de los aspectos gramaticales y vocabulario estudiados de forma no presencial en las sesiones presenciales.

Como ilustración de esta nueva metodología, a continuación presentamos una de las sesiones de flip teaching, correspondiente al segundo bloque temático del curso, cuyo tema central es Marketing, y que comprende las unidades 5 y 8 del libro de texto empleado en el curso Business Benchmark. La estructura de la actividad flip teaching es la siguiente:

A) Presentación de la Unidad 5: Breaking into the market

- El alumno debe comenzar por la actividad Getting started pag. 26 del libro del alumno. Esta actividad tiene como objetivo familiarizar al alumno con el vocabulario que utilizará en este bloque temático

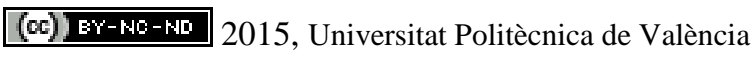

Congreso In-Red (2015) 
Flip-teaching aplicado al Inglés para la Gestión Empresarial: una nueva experiencia

- A continuación deberá realizar una lectura rápida del texto (skimming) Promoting Axe para tener una idea general del contenido del texto (no debe tardar mas de 2 minutos)

- Deberá realizar una lectura mas detallada del texto para hacer el ejercicio de opción múltiple y, al terminar, comprobar la consolidación del vocabulario realizando el ejercicio de emparejamieto de frases de la pag 28

- Como aspecto gramatical de esta unidad, se revisa el uso de verbos en infinitivo e -ing mediante el ejercicio de la pag 28. Para repasar la teoría, pueden consultar los usos en el libro Grammar workshop pag 42.

- Comprensión oral- el alumno deberá realizar el ejercico de comprensión oral Supermarket's own Brand de la pag 28, cuyo archivo de audio se ha subido a la plataforma PoliformaT

- Por último, el alumno realizará la lectura del texto Going to India and China donde trabajará sobre laidea principal de cada párrafo

B) Presentación de la Unidad 8 Being persuasive

- El alumno realizará una primera actividad de comprensión oral que le introduce el vocabulario de la unidad (pg 38)

- Ejercicio de lectura: The art of agreeing - en la que el alumno aprenderá el vocabulario para negociar mediante el ejercicio de relacionar frases y párrafos.

- Por último, ralizará un nuevo ejercicio de comprensión oral donde revisará el uso de las oraciones condicionales.

Adicionalmente a las actividades del libro, se le proporcionan al alumno enlaces a páginas de internet en las que puede practiccar aspectos relacionados con el vocabulario, la gramática o la comprensión oral que aparecen en la unidad.

A continuación mostramos algunas de las actividades tal y como se le mostrará al alumnno en su plataforma de PoliformaT.

La introducción de los objetivos del bloque y el trabajo a realizar se presentará al alumno, mediante una grabación con un Power Point utilizando el programa Screen cast omatic(free on-line screen recorder). Los objetivos de este bloque son (Fig.1): 


\section{BLOCK 2: MARKETING}

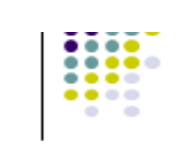

Topic: Marketing methods and strategies

- This unit is an introduction to marketing terms, and language to explore and discuss different marketing strategies and the thinking behind them.

- This unit gives students an introduction to marketing terms, and language to explore and discuss different marketing strategies and the thinking behind them.

\section{BLOCK 2: MARKETING}

\section{Topic: Marketing methods and strategies}

- This unit teaches spoken language for various stages in a business relationship:

- Meeting and greeting new customers/suppliers

- Making the sort of polite comments and questions that build a business relationship at outset.

- Asking about and talking about particular products, their characteristics and technical specifications

- Negotiating terms and conditions between customers and suppliers. 


\section{BLOCK 2: MARKETING}

\section{Topic: Marketing methods and strategies}

- This unit teaches spoken language for various stages in a business relationship:

- Meeting and greeting new customers/suppliers

- Making the sort of polite comments and questions that build a business relationship at outset.

- Asking about and talking about particular products, their characteristics and technical specifications

- Negotiating terms and conditions between customers and suppliers.

Fig. 1: Objetivos de la Sesión Flip-Teaching de IGE: Bloque 2: Marketing

Tras la presentación de los objetivos del bloque, se indican las actividades que el alumno debe realizar así como las estrategias comunicativas que irán adquirirendo con el desarrollo de dichas actividades. A continuación mostramos un ejemplo de una de las actividades de lectura (Fig. 2) que el alumno debe practicar en casa, en el que se indican las microdestrezas que se pretenden conseguir así como algunas técnicas de lectura:

\section{Unit 5. Breakinginto the market}

- Read the text on pg. 27. The Axe effect

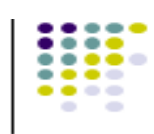

The Reading consists of:

- one text of 450-550 words

- six multiple-choice questions where you must choose the best answer, A,B,C or D.

\section{- Reading skills}

Here you test your ability to

- read in detail

- interpret opinions and ideas expressed in the text rather than facts. 


\title{
Unit 5. Breaking into the market
}

\author{
Reading pg. 27. The Axe effect
}

\section{Readinq techniques}

- Skim the text first to get a quick, general idea of what it says

- Read the stem of the firstquestion (the part before the A,B,C or D) and underline the key words.

- Find where the question is dealt with in the text. Read the four alternatives and find which one is correct.

- Remember: the words of the text will not repeat the words of the question. You will have to find the same idea expressed in a different way

- Make sure there is evidence in the text to support your answer

- The answers to the questions come in the same order in the text, so when you have answered one question, move on to the next part of the text to answer the next question.

Fig 2: Actividades, destrezas y estrategias de lectura

En esta sesión también se desarrollan las habilidades orales, tanto receptivas como productivas que serán esenciales para la sesión presencial de practical oral y debate. Aquí mostramos una de las actividades que el alumno practica en casa para adquirir las destrezas orales de cómo iniciar conversaciones o breaking the ice (Fig. 3):

\section{Unit 8.- Being persuasive}

ORAL SKILLS : Breaking the ice

- For many business people the hardest part of doing business is breaking the ice and making a small talk in English to build a relationship.

- Breaking the ice is often just a question of offering a series of positive or interested comments and questions which allow the other person to respond equally positively and give openings to more meaningful engagement.

The ideas in this exercise are typical things that British or American might say to break the ice

ACTIVITY: Listen to the passage and write down typical expressions you hear in each of these fragments:

1. How are you?

2. Did you have a good trip?

3. Lovely city, isn't' it?

4. Where are you staying?

5. now, can we show a few of our products?

6. Busy, isn't it?

7. Would you like a cup of coffee or a cup of tea before we get started?

Fig. 3. Actividad de comprensión oral

Para afianzar las estrategias de lectura y orales que se practican con las actividades del libro de texto, en la sesión de flip teaching también se les recomienda a los alumnos ejercicios complementarios de vocabulario, gramática, textos o vídeos relacionados con el te ma de

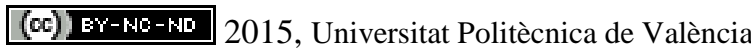

Congreso In-Red (2015) 
Marketing mediante enlaces directos a páginas de internet, como las que mostramos a continuación (Fig. 4):

\section{TOPIC: Presenting your company}

3- Put one word in each space to complete this extract from a presentation

Thank everyone (0) for coming. Today l'd like to (1)____ you a bit about our new company.

I'll be (2) __ so if you have any questions please wait and I'll be (3) ___ to

answer them at the end.

First of (4) __ how did we get the idea for Mobile Media? I met Steve my partner three months ago when he was working with Vodafone to ...

One other thing l'd like to point (5) already being used in China and Korea.

...Finally, l'd just like to (6) you this chart which $(7) \quad$ an overview of all the possibilities that this technology offers. We are really exited about the prospects for it and I hope I"ve been able to communicate some of that enthusiasm to you today. Thanks for (8) there any questions?

4. What comes next? Continue these presentation phrases using the prompts in brackets.

- Thank you /come/today) for coming today

- I'd like to begin by (tell/something/product)

- At this point I will quote our CEO: (great/product/not enough).

- Let's move on (look/sales).

- This chart (show/turnover/2007).

- Let's take a look (profits/last year)

- That brings me (end/presentation).

http://www.businessenglishsite.com/exercise_marketing1.html

Business English Exercise | Topic: Marketing Vocabulary 1

Designed to teach you the most common vocabulary/terms used in marketing. This exercise is meant for ESL (English as a second language) students, but will be useful to anyone wanting to learn this specialized terminology.

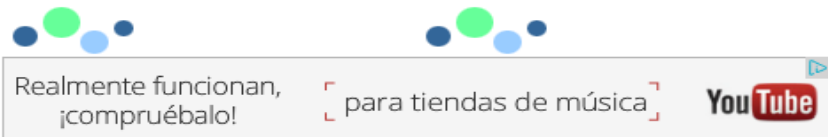

QUESTIONS:

(Choose the best response for each one)

1. Cell phone market

penetration

penalty

penance

2. I'm doing an

analysis

analyze

analytics

3. Every marketer should have an idea of how to build strong

Onames

Oideas

brands

http://www.englishmedialab.com/Quizzes/business/business\%20negotiation\%20words.htm

(c)) EY-NC-ND 2015, Universitat Politècnica de València 


\section{BUSINESS ENGLISH: Free Listening Comprehension Exercises} Exercise 1 (IBM Commercial)

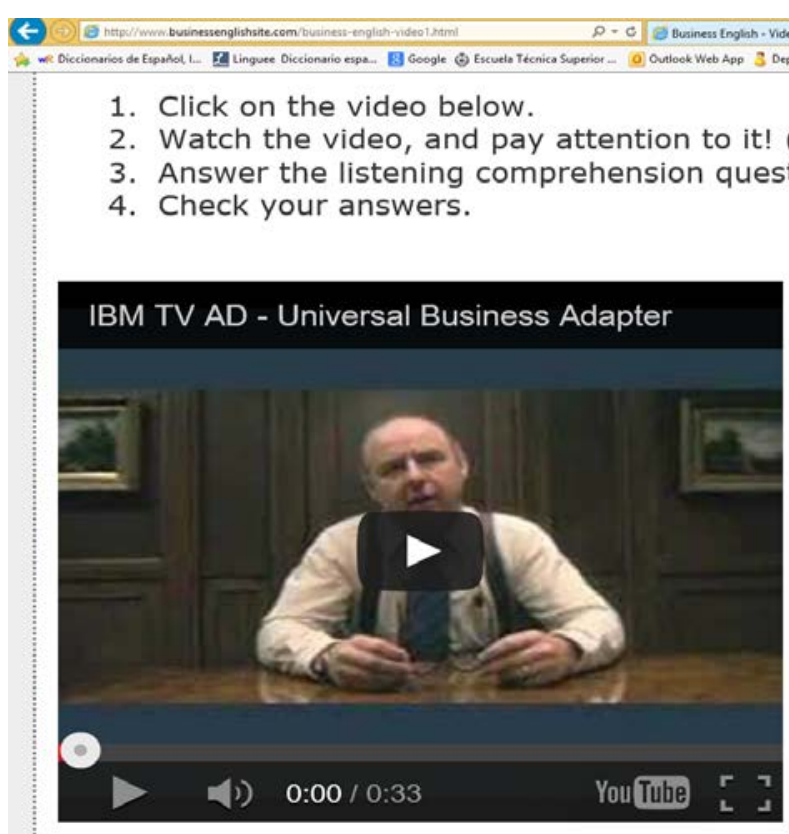

1. What does UBA stand for?:

- Universal Business Adaptor $\bigcirc$ "You-Be-Ay" $\bigcirc$ Universal Business Account

2. What does the UBA do?:

It connects "anything to everything" It connects a laptop to a desktop http://www.businessenglishsite.com/business-english-video1.html Fig. 4. Actividades complementarias

Aunque el tiempo total de la sesión de flip teaching variará según los conocimientos de cada alumno, la duración prevista es de 4 horas, que el estudiante puede ir distribuyendo a lo largo de dos semanas.

Así, en la sesión presencial de control de 50 minutos se revisará el vocabulario y expresiones más importantes relacionadas con Marketing y se consolidarán los puntos gramaticales, ya que en la Práctica de Laboratorio presencial de 2 horas, el alumno tendrá que realizar principalmente actividades de expresión oral, en las que deberá poner en práctica las destrezas comunicativas, léxicas y semánticas aprendidas en las tareas de flip teaching. Las actividades orales correspondientes a este bloque 2 son:

1. Un role play sobre el lanzamiento de un nuevo producto

2. Preparar una breve presentación sobre una cadena de tiendas que conozacan bien

\section{(cc)) EY-NC-ND 2015, Universitat Politècnica de València}

Congreso In-Red (2015) 
Flip-teaching aplicado al Inglés para la Gestión Empresarial: una nueva experiencia

3. Negociar con los demás grupos las mejores técnicas de mercado para promocionar un producto

4. Redactar un breve resumen con las conclusiones

De esta manera, las clases presenciales serán totalmente prácticas e interactivas , el alumno tendrá la oportunidad de adquirir técnicas de expresión oral realizando presentaciones de los temas trabajados en casa, técnicas de trabajo en equipo realizando debates que simulan situaciones empresariales que le acerquen al mundo laboral para el que le preparan sus estudios de ADE.

\section{Resultados}

Si bien, como hemos comentado, es una iniciativa que pretendemos implementar en el próximo curso académico, podemos adelantar algunos de los resultados que esperamos lograr con la introducción de esta nueva metodología, de acuerdo con los comentarios de los distintos estudios consultados en la literatura existente sobre el tema (Bergmann et al., 2011, Stone, 2012, Sánchez, 2013, Gutiérrez et al., 2013, Shapiro, 2013, Sánchez et al., 2014, Rincón, 2015) y que resumimos a continuación:

- Crear un ambiente de aprendizaje centrado en el estudiante

- Mejora significativa del ambiente de trabajo en el aula

- $\quad$ Cambio del papel del profesor que se convierte en un guía en el aprendizaje

- $\quad$ Flexibilización y personalización del aprendizaje permitiendo que cada alumno realice las actividades en función de su capacidad y dominio del idioma

- El uso de la tecnología permitirá un acceso fácil a los materiales de aprendizaje en cualquier momento y lugar

- Mejora sustancial de las habilidades y estrategias comunicativas de los alumnos

\section{Conclusiones}

Es necesario introducir nuevas metodologías que estimulen el aprendizaje de los estudiantes. El uso de flip-teaching, que combina las nuevas tecnologías con los sistemas de enseñanza convencionales, mejora el rendimiento de los alumnos ya que permite utilizar el tiempo en clase para aplicar las enseñanzas teóricas que los alumnos pueden estudiar individualmente en casa.

Esta nueva metología creemos que puede resultar particularmente útil para el conocimiento de idiomas ya que la mejor forma de adquirir competencia lingüística es a través del desarrollo de estrategias comunicativas a través de la interacción y la participación activa de los alumnos. 
La propuesta que presentamos supone la optimización del tiempo asignado en los planes de estudio a la asignatura de Inglés para la Gestión Empresarial (IGE) nivel B1de modo que los alumnos puedan alcanzar el Nivel B2 necesario para graduarse al finalizar sus estudios.

A pesar de las ventajas nombradas, habrá que esperar a tener los resultados reales para poder valorar los beneficios de esta nueva metodología. Así, en un futuro trabajo compararemos el rendimiento de los estudiantes que han cursado la asignatura de IGE siguiendo el sistema convencional de clases presenciales con los resultados obtenidos en el grupo flip teaching para comrpobar las ventajas de la enseanza inversa.

Podríamos concluir que en educación no hay fórmulas mágicas que se adapten a todas las circunstancias y situaciones, sino que se trata de aprovechar las potencialidades de todos los recursos, quedarnos con lo que ofrecen de bueno para conseguir nuestros objetivos. Las clases invertidas representan una posibilidad más que la tecnología pone a nuestro alcance, una ayuda para que el profesor se convierta en un facilitador del aprendizaje, una nueva visión a tener en cuenta (Sánchez et al., 2014)

\section{Referencias}

BAKER, J. W. (2000). "The "classroom flip": Using web course management tools to become the guide by the side" en 11th International Conference on College Teaching and Learning, Jacksonville, FL., pp. 28-34

BERGMANN, J. y SAMS, A. (2012). "Flip Your Classroom: Reach Every Student in Every Class Every Day" en International Society for Technology in Education. ASCD Pub. USA., pp. 12-22

BERGMANN,.J. OBERMYER, G. y WILLIE, B. (2011). "The flipped class: What it is and What it is not. The Daily Riff". <http://www.thedailyriff.com/articles/the-flipped-classconversation-689.php> [Consulta: 8 de febrero 2015].

BERLIN DECLARATION (2001). Language Studies in Higher Education: A Key Contribution to European Integration. <http://userpage.fu-berlin.de/elc/docs/ BDeclEN.pdf> [Consulta: 10 de marzo de 2012].

DEMETRY, Ch. (2010). "Work in Progress - An Innovation Merging."Classroom Flip” and Team-Based Learning." En 40th ASEE/IEEE Frontiers in Education Conference. IEEE October 27 - 30, 2010, Washington, DC. Pp. 43-56.

DOCUMENTO MARCO UPV (2008). Documento de Diseño de titulaciones. Documento marco UPV, fecha febrero 2008.

EIU (2011). Estrategia de internacionalización del sistema universitario español Estrategia universidad 2015, EIU septiembre 2011. 
Flip-teaching aplicado al Inglés para la Gestión Empresarial: una nueva experiencia

<http://cms.ual.es/idc/groups/public/@vic/@vinternacional/

documents/documento/relintinter3.pdf> [Consulta: 2 febrero de 2014].

EUROPEAN LANGUAGE COUNCIL (2005) European Network for the Promotion of Language Learning Among All Undergraduates. $<$ http://www.google.es/url?sa=t\&rct=j\&q=\&esrc $=$ s\&source $=$ web\&cd $=2 \& v e d=0 C C 4 Q F j A$ B\&url=http\%3A\%2F\%2Fuserpage.fuberlin.de\%2F enlu\%2Fdownloads\%2F050519_ExpressionofInterest.doc\&ei=er1gVYmXL cT6UPzwgLAH\&usg=AFQjCNF2znrzeJih1CZ8ACDniTFr3DLO2Q\&sig2=1T_SwOSnZt R-zUABqH9UFQ\&bvm=bv.93990622,d.ZGU> [Consulta: 2 febrero de 2014].

FULTON, K. (2012). "Upside down and inside out: Flip Your Classroom to Improve Student Learning” en Learning \& Leading with Technology 39/8, pp.12-17.

GUTIÉRREZ, I., CASTAÑEDA, L. y SERRANO, J. (2013). "Más allá de la Flipped Classroom: "dar la vuelta a la clase" con materiales creados por los alumnos" en II Congreso Internacional Educación Mediática y Competencia Digital. Barcelona, España. http://dspace.uma.es/xmlui/bitstream/handle/10630/7821/comunicacion.pdf?sequence=6 > [Consulta: 2 febrero de 2014].

HAUG, G. y VILALTA, J.M. (2011). "La internacionalización de las universidades, una estrategia necesaria” en Studia XXI. Fundación Europea Sociedad y Educación. $<$ http://www.celelc.org/archive/conference/Conference_documents/english_0.pdf?1370253 481> [Consulta: 2 de febrero de 2014].

LASRY, N., DUGDALE, M y CHARLES, E. (2014). "Just in Time to Flip Your Classroom” en Phys. Teach. 52, 34 < http://dx.doi.org/10.1119/1.4849151. > [Consulta: 15 de enero de 2015].

PAPPANO, L. (2012). "The Year of the MOOC” en The New York Times, November 2. Internet: <http://www.nytimes.com/2012/11/04/education/edlife/massive-open-onlinecourses-are-multiplying-at-a-rapid-pace.html > [Consulta: 4 de febrero de 2015].

RINCÓN ORTIZ, J.C. (2015). "Flipped Clasroom. Caso: Proyectos Básicos de Ingeniería de Ríos” en Luisa Casadei Carniel (Ed.) Innovación educativa apoyada por las TIC en la Universidad Centroccidental Lisandro Alvarado. Biblioteca Virtual de la UCLA: Venezuela 2015, pp 9-24.

SAMS, A y BERGMANN, J. (2013). "Flip Your Students' Learning” en Educational Leadership 70/6 pp16-20.

SÁNCHEZ, J., RUIZ, J. y SÁNCHEZ, E. (2014). "Las clases invertidas: beneficios y estrategias para su puesta en práctica en la educación superior” en XIX Congreso Internacional de Tecnologías para la Educación y el Conocimiento 
<http://dspace.uma.es/xmlui/bitstream/handle/10630/7821/

comunicacion.pdf?sequence $=6>$ [Consulta: 4 de febrero de 2015] .

SÁNCHEZ, M. (2013). "Memoria final del proyecto Flipped TIC: diseño de una experiencia Flipped Classroom en el aula. Convocatoria experiencias de Innovación Educativa. Curso 2012/2013”. Facultades de la Universidad de Murcia. $<$ http://digitum.um.es/jspui/bitstream/10201/35812/1/

Memoria\%20final\%20Flipped\%20TIC.pdf.> [Consulta: 4 de febrero de 2015].

SHAPIRO, M. (2013). "Flipped classroom turns traditional teaching upside down". $<$ http://www.stltoday.com/suburban-journals/metro/education/flipped-classroomturnstraditional-teaching-upside-down/article_a6497f82-efb3-5a62-88edee72c2ac873c.html>. [Consulta: 8 de febrero de 2015].

STONE, B. (2012). "Flip your classroom to increase active learning and student engagement” en 28th annual conference on Distance Teaching \& Learning $<$ http://www.uwex.edu/disted/conference/resource_library/search_detail.cfm?presid=56511 $>$. [Consulta: 15 de enero de 2015]. 